\title{
Editorials
}

\section{COVID-19 highlights health promotion and chronic disease prevention amid health disparities}

With no identified vaccine and no clearly effective treatment, COVID-19 healthcare systems around the world have placed an extreme focus on strategies to prevent transmission of COVID-19 and prioritising, triaging, and treating these patients. Many patients have been told not to come to the doctor unless they have flu-like illness. By focusing almost exclusively on strategies to decrease transmission of the COVID-19 pandemic are we missing the important role of primary care in protecting the health of those with chronic disease? Moreover, by de-emphasising chronic disease management are we potentially increasing the impact of COVID-19 and the likelihood of severe outcomes?

\section{KEY ROLE OF AMBULATORY CARE AND CHRONIC DISEASE MANAGEMENT}

Chronic disease appears to play a huge role in COVID-19 severity and outcomes. It has been estimated that many US adults are at increased risk for COVID-19 hospitalisation because of ambulatory care sensitive conditions (ACSCs), such as existing cardiovascular disease, diabetes, respiratory disease, and hypertension. ${ }^{1}$ People with underlying medical conditions such as heart disease and diabetes were hospitalised six times more than otherwise healthy individuals infected with COVID-19 during the first 4 months of the pandemic, and were 12 times more likely to die. ${ }^{2}$ Similar health profiles were reported by mid-April 2020 in the UK. Among 20000 hospital COVID-19 inpatients, $>75 \%$ had pre-existing obesity, diabetes mellitus, chronic heart, lung, or other disease, and these were risk factors for death. ${ }^{3}$

Primary care plays a key role in health promotion and disease prevention, particularly with ACSCS. ACSCs and their management are dependent on access to appropriate care, and in particular primary care. Too severe of a focus on infectious disease may be counterproductive for the

. by de-emphasising chronic disease management are we potentially increasing the impact of COVID-19 and the likelihood of severe outcomes?"

general population of patients. A recent survey in the US showed that $48 \%$ of households skipped or postponed medical care due to the COVID-19 pandemic.4 Appropriate primary care seems to play a positive role in decreasing the impact of COVID-19 and attention to conditions other than just flu-like illness may pay positive dividends. However, the COVID19 pandemic has not just highlighted the value of primary care and chronic disease prevention, it has also emphasised existing health disparities, explicitly in ACSCs.

\section{NEGLECT OF CHRONIC DISEASE} MANAGEMENT AND HEALTH DISPARITIES Health disparities in ACSCs were pervasive even before the pandemic. The National Institute of Minority Health and Health Disparities (NIMHD) and others have pointed to the disproportionate burden of COVID-19 on minorities and the need for a better understanding of the reasons for this. ${ }^{5.6}$ One reason may be the distribution of ACSCs in minority populations and their ability to control their chronic disease both before and during the COVID-19 pandemic. The effects of COVID-19 on the health of racial and ethnic minority groups suggest a more magnified, disproportionate burden of illness and death. In analyses across 14 states in the US and one in Georgia focusing on hospitalisations, black populations appear to be disproportionately affected by COVID-19.1.7 More than 33\% of those hospitalised were black; while black ethnicity constitutes only $13 \%$ of the US population. Of all patients hospitalised for COVID-19, about $90 \%$ had at least one of

\section{"The pandemic crisis is exacerbating pre-existing racial disparities in ambulatory care sensitive conditions, which may have contributed to more severe cases of and higher death rates due to COVID-19.}

the ACSCs. ${ }^{1}$ The greater odds of hospital admission among the black population may indicate that these individuals have more advanced or severe illness at the time of presentation for medical care. It is likely that patients are being influenced by messages and situations that either result in barriers to timely access to care or situations in which patients view delaying care as the preferred strategy. ${ }^{8}$

Similar disparities are seen in the UK, with black and Asian minority groups having 2-4 times the risk of hospitalisation and death.? Ethnic minority communities in the UK are also likely to be at increased risk of poorer outcomes once they acquire the infection. Some comorbidities, such as diabetes, which increase the risk of poorer outcomes from COVID-19, are more common among black and Asian populations than among the white population in the UK. The pandemic crisis is exacerbating preexisting racial disparities in ACSCs, which may have contributed to more severe cases of and higher death rates due to COVID-19.

\section{PRIMARY CARE IN LOW INCOME COUNTRIES}

This impact on primary care and care for chronic disease by the emphasis on COVID19 is not only a problem in places like the US and the UK. In low income countries (LIC) where the healthcare system is already weak, COVID-19 places a heavy burden and fractures care for chronic disease. For example in Haiti, fear of contracting COVID19 and the implementation of quarantine places a significant impact on careseeking for chronic diseases. Moreover stigmatisation of people with COVID-19 impedes care. Hospitals treating patients with COVID-19 in Haiti have been physically attacked. ${ }^{10}$ Even though many LICs like Haiti have a high death rate due to infectious disease there is still considerable chronic disease, with the majority of people dying from chronic disease. ${ }^{11}$ Thus, restricting care to COVID-19 will only increase severe disease for patients with chronic disease, 


\section{"In low income countries where the healthcare system is already weak, COVID-19 places a heavy burden and fractures care for chronic disease.}

and potentially increase the severity of COVID-19 outcomes because of the link between underlying ACSCs and COVID-19 outcomes.

\section{WHERE DO WE GO FROM HERE?}

One disease should not define the entire health system. Access to care for conditions other than COVID-19-like illness need not be lost. In fact, based on the evidence showing the link between chronic disease and COVID-19 outcomes it may be prudent to improve access to care systems for those with chronic disease. Additionally, the COVID-19 pandemic highlights the need for culturally competent health promotion and disease prevention programmes for noncommunicable diseases.

\section{Arch G Mainous III,}

Professor, Department of Health Services Research Management and Policy, University of Florida, Department of Community Health and Family
Medicine, University of Florida, Gainesville, FL, US.

\section{Sonia Saxena,}

Professor, Faculty of Medicine, School of Public Health, Imperial College London, London, UK.

\section{Valery M Beau de Rochars,}

Assistant Professor, Department of Health Services Research, Management and Policy, University of Florida; Emerging Pathogens Institute, University of Florida, Gainesville, FL, US.

\section{Donald Macceus,}

Staff physician, Zanmi Lasante, Partners in Health, Haiti.

\section{Provenance}

Commissioned; not externally peer reviewed.

\section{Competing interests}

The authors have declared no competing interests.

DOI: https://doi.org/10.3399/bjgp20X711785

\section{ADDRESS FOR CORRESPONDENCE}

\section{Arch G Mainous III}

Department of Health Services Research,

Management and Policy, University of Florida, Health Sciences Center, PO Box 100195, Gainesville, FL, US.

\section{Email: arch.mainous@ufl.edu}

\section{REFERENCES}

1. Garg S, Kim L, Whitaker M, et al.

Hospitalization rates and characteristics of patients hospitalized with laboratory-confirmed coronavirus disease 2019 - COVID-NET,

14 States, March 1-30, 2020. MMWR Morb Mortal Wkly Rep 2020; 69(15): 458-464

2. Stokes EK, Zambrano LD, Anderson KN, et al Coronavirus disease 2019 case surveillance - United States, January 22-May 30, 2020. MMWR Morb Mortal Wkly Rep 2020; 69(24): 759-765

3. Docherty AB, Harrison EM, Green CA, et al Features of 20133 UK patients in hospital with covid-19 using the ISARIC WHO Clinical Characterisation Protocol: prospective observational cohort study. BMJ 2020; DOI: 10.1136/bmj.m1985.

4. Hamel L, Kearney A, Kirzinger A, et al. KFF health tracking poll - May 2020. Impact of coronavirus on personal health, economic and food security, and medicaid. 2020. https:// www.kff.org/report-section/kff-health-trackingpoll-may-2020-health-and-economic-impacts laccessed 8 Jul 2020).

5. Webb Hooper M, Nápoles AM, Pérez-Stable EJ. COVID-19 and racial/ethnic disparities. JAMA 2020; DOI: 10.1001/jama.2020.8598.

6. Yancy CW. COVID-19 and African Americans. JAMA 2020; DOI: 10.1001/jama.2020.6548.

7. Gold JAW, Wong KK, Szablewski CM, et al. Characteristics and clinical outcomes of adult patients hospitalized with COVID-19 - Georgia, March 2020. MMWR Morb Mortal Wkly Rep 2020; 69(18): 545-550.

8. Azar KMJ, Shen Z, Romanelli RJ, et al. Disparities in outcomes among COVID-19 patients in a large health care system in California. Health Aff 2020; DOI: 10.1377/ hlthaff.2020.00598.

9. Public Health England. Disparities in the risk and outcomes of COVID-19. 2020. https:// assets.publishing.service.gov.uk/government/ uploads/system/uploads/attachment_data/ file/892085/disparities review.pdf laccessed 8 Jul 2020).

10. Rouzier V, Liautaud B, Deschamps MM. Facing the monster in Haiti. N Engl J Med 2020; DOI: 10.1056/NEJMc2021362

11. World Health Organization. Noncommunicable diseases country profiles 2018. https://wnw. who.int/nmh/countries/en laccessed 8 Jul 2020). 